\title{
The rice XA21 ectodomain fused to the Arabidopsis EFR cytoplasmic domain confers resistance to Xanthomonas oryzae pv. oryzae
} \author{
Benjamin Schwessinger \\ Pamela C Ronald ${ }^{\text {Corresp. }} 1$ \\ ${ }^{1}$ Department of Plant Pathology and the Genome Center, University of California, Davis, Davis, CA, United States \\ 3 Research School of Biology, Australian National University, Acton, Australia \\ Corresponding Authors: Benjamin Schwessinger, Pamela C Ronald \\ Email address: benjamin.schwessinger@anu.edu.au, pcronald@ucdavis.edu
}

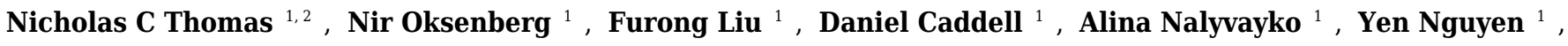

Rice (Oryza sativa) plants expressing the XA21 cell surface receptor kinase are resistant to Xanthomonas oryzae pv. oryzae (Xoo) infection. We previously demonstrated that expressing a chimeric protein containing the EFR ( ELONGATION FACTOR TU RECEPTOR) ectodomain and the XA21 endodomain (EFR:XA21) in rice does not confer robust resistance to $X_{00}$. To test if the XA21 ectodomain is required for $X o 0$ resistance, we produced transgenic rice lines expressing a chimeric protein consisting of the XA21 ectodomain and EFR endodomain (XA21:EFR) and inoculated these lines with Xoo. We also tested if the XA21:EFR rice plants respond to a synthetic sulfated 21 amino acid derivative (RaxX21-sY) of the activator of XA21-mediated immunity, RaxX. We found that five independently transformed XA21:EFR rice lines displayed resistance to Xoo as measured by lesion length analysis, and showed that five lines share characteristic markers of the XA21 defense response (generation of reactive oxygen species and defense response gene expression) after treatment with RaxX21-sY. Our results indicate that expression of the XA21:EFR chimeric receptor in rice confers resistance to $X_{00}$. These results suggest that the endodomain of the EFR and XA21 immune receptors are interchangeable and the XA21 ectodomain is the key determinant conferring robust resistance to Xoo. 
1 The Rice XA21 Ectodomain Fused to the Arabidopsis EFR Cytoplasmic Domain

2 Confers Resistance to Xanthomonas oryzae pv. oryzae

3 Nicholas Thomas ${ }^{1,3^{*}}$, Nir Oksenberg ${ }^{1 *}$, Furong Liu ${ }^{1}$, Daniel Caddell ${ }^{1}$, Alina Nalyvayko ${ }^{1}$,

4 Yen Nguyen ${ }^{1}$, Benjamin Schwessinger ${ }^{1,2, \dagger}$ and Pamela Ronald ${ }^{1, \dagger}$

5 'Department of Plant Pathology and the Genome Center, University of California, Davis,

6 Davis, California, United States of America

7 2The Australian National University, Research School of Biology, Acton ACT 2601,

8 Australia.

9 3Department of Plant Pathology and Microbiology, University of California, Riverside,

10 Riverside, California 92521, USA

$11{ }^{*}$ These authors contributed equally to this work and are shown in reverse alphabetical

12 order

$13+$ These authors are co-corresponding authors

\section{Abstract}

Rice (Oryza sativa) plants expressing the XA21 cell surface receptor kinase are

17 resistant to Xanthomonas oryzae pv. oryzae (Xoo) infection. We previously demonstrated that expressing a chimeric protein containing the EFR (EELONGATION FACTOR TU RECEPTOR) ectodomain and the XA21 endodomain (EFR:XA21) in rice does not confer robust resistance to Xoo. To test if the XA21 ectodomain is required for Xoo resistance, we produced transgenic rice lines expressing a chimeric protein consisting of the XA21 ectodomain and EFR endodomain (XA21:EFR) and inoculated 
24 sulfated 21 amino acid derivative (RaxX21-sY) of the activator of XA21-mediated 25 immunity, RaxX. We found that five independently transformed XA21:EFR rice lines displayed resistance to Xoo as measured by lesion length analysis, and showed that 27 five lines share characteristic markers of the XA21 defense response (generation of

28

reactive oxygen species and defense response gene expression) after treatment with RaxX21-sY. Our results indicate that expression of the XA21:EFR chimeric receptor in rice confers resistance to Xoo. These results suggest that the endodomain of the EFR and XA21 immune receptors are interchangeable and the XA21 ectodomain is the key determinant conferring robust resistance to Xoo.

\section{Introduction}

Plant cell surface immune receptors confer defense against pathogen infection. Cell surface mediated immunity in plants is mainly conferred by receptor like proteins (RLPs) and receptor like kinases (RLKs) that recognize pathogen associated molecular patterns (PAMPs) (Jones \& Dangl, 2006; Macho \& Zipfel, 2014). Three well-studied cellsurface RLKs that confer resistance to bacterial pathogens include FLAGELLIN SENSING2 (FLS2; At5G6330) (Gómez-Gómez \& Boller, 2000), EF-TU RECEPTOR (EFR; At5g20480) (Zipfel et al., 2006) from Arabidopsis and XA21 (U37133) from Oryza longistaminata (Song et al., 1995). The identification of the microbial molecules recognized by these three receptors have enhanced in depth characterization of their functional properties. The FLS2 receptor binds the flg22 peptide derived from bacterial Flagellin (Felix et al., 1999; Gómez-Gómez \& Boller, 2000; Chinchilla et al., 2006). EFR recognizes the elf18 peptide derived from the bacterial Elongation Factor Thermo- 
47 unstable protein (EF-Tu) (Kunze et al., 2004; Zipfel et al., 2006). XA21 recognizes the 48 sulfated RaxX (required for activation of $\underline{X}$ a21-mediated immunity $\underline{X}$ ) protein produced by Xoo (Pruitt et al., 2015). Although these receptors specifically recognize different 50 molecules, they share similar domain structures including ectodomains containing 51 leucine rich repeats and endodomains containing intracellular kinases of the non52 arginine aspartate (non RD) class (Dardick and Ronald, 2006).

54 that the nature of the endodomains is the primary determinant dictating the specific 55 disease resistance outcome. For example, studies of a chimeric receptor generated by 56 fusion of the Arabidopsis BRASSINOSTEROID-INSENSITIVE1 (BRI1) receptor, which 57 recognizes brassinosteroid hormones, to the XA21 endodomain (BRI1:XA21) (Li \& Chory, 1997) indicated that the chimeric receptor could be activated by brassinosteroid treatment. Rice cells expressing BRI1:XA21 (NRG-1 in the original publication) and treated with brassinosteroid initiated cell death, produced ROS, and expressed stressrelated genes. The stress-related symptoms were attributed to the activation of the XA21 endodomain because the full-length BRI1 receptor does not induce the same stress-related symptoms as BRI1:XA21 (He et al., 2000). These results suggested that the XA21 endodomain was activated upon BRI1 recognition of brassinosteroid and that the specific type of response was most consistent with the response mediated by the XA21 endodomain and not the BRI1 ectodomain.

Another chimera study compared the responses of receptors consisting of ectodomain and endodomain exchanges between EFR and WAK1. WAK1 recognizes oligogalacturonides (OGs) released from damaged plant cell walls (Decreux \& 
70 Messiaen, 2005; Decreux et al., 2006; Cabrera et al., 2008). Elf18 treated wild-type 71 plants and OG treated plants expressing a WAK1:EFR (WEG) chimeric RLK both 72 produced ROS, ethylene and expressed the EFR-induced genes (At3g22270 and 73 At4g37640) while EFR:WAK1 (EWAK) expressing plants did not. Instead, EWAK plants 74 retained WAK1-like responses by producing ROS but not ethylene in response to OGs 75 (Ferrari et al., 2008; Brutus et al., 2010). WEG and EWAK responses were therefore 76 most consistent with the response conferred by the respective endodomain portion of 77 each fusion protein. Another study showed that fusing the XA21 endodomain to the 78 fungal chitin receptor like protein CEBiP (Kaku et al., 2006) (CRXA-1, and CRXA-3 in 79 the original publication) conferred a more robust immune response to fungal infection by 80 Magnaporthe oryzae than when expressing or overexpressing CEBiP alone (Kishimoto 81 et al., 2010). These results suggested that the XA21 endodomain was responsible for 82 conferring the enhanced immune response to $M$. oryzae. Together, these studies 83 indicate that the endodomain of several immune receptors dictate the specific signaling 84 events that lead to disease resistance in whole plants or defense responses in plant 85 cells. These studies also suggest chimeras carrying the XA21 endodomain, when 86 treated with the appropriate ligand, can initiate an immune response similar to that 87 mediated by the full-length receptor XA21.

To further explore the function and specificity of the XA21 endodomain and 89 ectodomain, we previously generated transgenic rice lines expressing EFR, tagged with 90 green fluorescent protein (EFR:GFP), or a chimeric EFR:XA21 protein, consisting of the 91 EFR ectodomain and the XA21 transmembrane and intracellular domain, tagged with 92 GFP (EFR:XA21:GFP). The maize ubiquitin promoter drove expression for both 
EFR:GFP and EFR:XA21:GFP transgenes (Schwessinger et al., 2015a). Both

94 EFR:GFP and EFR:XA21:GFP rice plants were susceptible to Xoo strain PXO99A and

95

96

conferred partial resistance to weakly virulent strains, which suggested EF-Tu from Xoo was still recognized by EFR:GFP rice (Schwessinger et al., 2015a). These studies suggested that although both receptors were capable of recognizing EF-Tu, they were still unable to initiate a robust immune response to PXO99A. As noted in the paper's discussion, these results were counterintuitive based on earlier domain swap studies that indicated that the endodomain dictates immune signaling and disease resistance (He et al., 2000; Brutus et al., 2010; Albert et al., 2010; Kishimoto et al., 2010).

Although it is unclear why the EFR and EFR:XA21 study conflicted with findings from previous chimeric receptor studies, there are several possibilities to explain these discrepancies. In the case of the EFR:WAK1 and WAK1:EFR study, it could be that the type of kinase domain dictated the distinct signaling mediated by each chimeric receptor because the WAK1 and EFR kinase domains belong to different kinase classes. The WAK1 kinase domain contains an arginine (R) aspartate (D) motif while the EFR kinase domain is non-RD, as described above. The non-RD kinases are almost always associated with immune responses in plants and animals and are likely regulated differently than RD kinases (Dardick \& Ronald, 2006; Ronald \& Beutler, 2010; Dardick, Schwessinger \& Ronald, 2012). Thus, the presence of the non-RD domain may dictate an immune response when appropriately activated and the presence of the RD domain may specify a WAK1-like response.

For both BRI1:XA21 and CEBiP:XA21 studies, it is possible that the origin of the kinase domain from XA21 was less important than the fact that the kinase belonged to 
116 the non-RD class. For example, it is unclear if fusing BRI1 or CEBiP to other non-RD

117 kinases, such as the kinases from EFR or OsFLS2, would have produced similar results

118 (Takai et al., 2008).

119 Previous studies have shown that the XA21 ectodomain plays a critical role in the 120 immune response. For example, the Xa21D paralog, which lacks a transmembrane and 121 intracellular domain, confer partial resistance to Xoo (Wang et al., 1998). Unlike Xa21, 122 Xa21D only encodes an ectodomain that is nearly identical to the XA21 ectodomain, 123 differing only in 15 amino acid residues compared to the XA21 ectodomain. Similarly, 124 expression of a catalytically inactive variant of XA21, carrying a mutation in the catalytic domain of the kinase (K736E), in rice maintained partial resistance to Xoo (Andaya \& Ronald, 2003b). Together, these studies indicate that the XA21 ectodomain is sufficient to confer partial resistance to Xoo, even in the absence of a functional kinase domain.

To further explore the function and importance of the XA21 ectodomain, we 129 generated transgenic rice lines expressing a chimeric protein containing the XA21 ectodomain fused to the EFR transmembrane and intracellular domain, tagged with GFP (XA21:EFR:GFP) (Holton et al., 2015). We found that XA21:EFR:GFP rice display robust resistance to Xoo strain PXO99A. We also show that XA21:EFR:GFP was specifically activated by RaxX as measured by bacterial infection, defense response gene expression and ROS production (Pruitt et al., 2015; Schwessinger et al., 2015b;

Wei et al., 2016). These results indicate that the XA21 ectodomain and its recognition of

\section{Materials and Methods}


Plant material and methods

140 Rice seeds were germinated on water-soaked filter paper for $5-7$ days at $28^{\circ} \mathrm{C}$ and then

141 transplanted into 2.6-liter pots. Plants were grown in an approximately 80/20

142 (sand/peat) soil mixture in an environmentally-controlled greenhouse with temperature 143 set between $28-30^{\circ} \mathrm{C}$ with $75-80 \%$ humidity.

145 Transgenic rice production

146 The Xa21:EFR:GFP (XA21 aa residues 1-650 fused to EFR aa residues 650-1031)

147 binary vector used in rice transformation was described previously (Holton et al., 2015).

148 Transgenic Kitaake plants expressing the Xa21:EFR:GFP transgene, under control of 149 the maize ubiquitin promoter, were generated by the UC Davis Plant Transformation 150 Facility as described previously (Hiei et al., 1994). pCAMBIA1300 binary vectors 151 carrying the Xa21:EFR:GFP construct were transformed into Kitaake calli by 152 Agrobacterium-mediated transformation. Regenerated plants were selected on 153 hygromycin. The presence of the transgene was confirmed in each generation by PCR 154 using transgene specific primers (Table S1).

155

156 Bacterial infection of rice plants

157 Xoo isolates PXO99A, PXO99A $\triangle \operatorname{rax} X$, and PXO99A $\Delta r a x X(\operatorname{rax} X)$ (Pruitt et al., 2015)

158 were plated on peptone sucrose agar plates for 3 days. Xoo was suspended in water to 159 approximately $5 \times 10^{8}$ colony forming units $(\mathrm{CFU}) / \mathrm{mL}$ for inoculation. Greenhouse160 grown plants were transported into environmentally controlled growth chambers at the 4 161 week-old stage. Chamber conditions were set to $26^{\circ} \mathrm{C}, 85 \%$ humidity with $12 \mathrm{~h}$ light/dark 
162 cycles. Plants were acclimated to the chamber conditions for 2-3 days before scissor

163 inoculation (Kauffman et al., 1973).

164

165 Segregation analysis

166 The presence of each transgene was identified using PCR genotyping using genomic

167 DNA templates and transgene-specific primers (Table S1). Chi-square tests were used 168 to determine possible multiple transgene insertions.

169

170

Gene expression analysis by $q R T-P C R$

171 Total RNA was extracted from detached leaves frozen in liquid nitrogen and powdered using a

172 Qiagen tissuelyser. RNA was extracted from powdered tissue using TRI Reagent and

173 precipitated with isopropanol. RNA was DNase treated using the TURBO DNase kit from Life

174 Technologies. RNA concentrations were normalized to the lowest sample concentration in each

175 experiment. cDNA was synthesized from $2 \mu \mathrm{g}$ of total RNA using the High Capacity cDNA

176 Reverse Transcription Kit by Life Technologies. Gene expression changes were determined by

$177 \Delta \Delta$ Ct method (Livak \& Schmittgen, 2001) normalizing gene expression to Actin

178 (LOC_Os03g50885) and using mock treated samples as the reference for stress gene

179 expression. Quantitative real time PCR (qRT-PCR) was performed using a Bio-Rad

180 CFX96 Real-Time System coupled to a C1000 Thermal Cycler (Bio-Rad) using the Bio-

181 Rad SsoFast EvaGreen Supermix. qRT-PCR primer pairs used are described in Table

182 S1. qRT-PCR reactions were run for 40 cycles with annealing and amplification at $62^{\circ} \mathrm{C}$

183 for $5 \mathrm{sec}$ and denaturation at $95^{\circ} \mathrm{C}$ for $5 \mathrm{sec}$. Single melting curves were observed for all

184 primer pairs used indicating negligible off-target amplification. 
Western Blot Analysis for Protein expression

Anti-GFP (Santa Cruz Biotech) was used to detect EFR:GFP, EFR:XA21:GFP,

XA21:GFP and XA21:EFR:GFP. Secondary anti-mouse antibodies (Santa Cruz

189

Biotech) conjugated to horseradish peroxidase were used in combination with chemiluminescence substrates (Thermo) to detect proteins on a Biorad ChemiDoc.

Reactive oxygen species production

193 Leaves of 3- to 4-week-old rice plants were cut longitudinally along the mid vein and

194 then into 1 to $1.5 \mathrm{~mm}$ thick pieces. Leaf pieces were floated on sterile water overnight.

195 The following morning, two leaf pieces were transferred into one well of a 96-well white

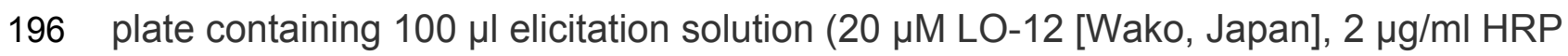

197 [Sigma]). 500 nM of elf18 (Escherichia coli) or RaxX21-sY peptides were used for 198 treatments. ROS production was measured for 0.5 s per reading with a high sensitivity plate reader (TriStar, Berthold, Germany).

200

201

\section{Results}

202 Transgenic rice expressing the XA21:EFR chimeric receptor display robust 203 resistance to Xoo.

We produced transgenic rice lines expressing an Xa21:EFR:GFP chimeric 205 construct to test whether the XA21 ectodomain confers resistance to Xoo when fused to

206 the EFR cytoplasmic domain. This construct encodes the XA21 ectodomain (XA21

207 residues 1-650) fused to the EFR transmembrane, juxtamembrane and cytoplasmic 
208 domain (EFR residues 651-1031) with a carboxyl-terminal GFP fusion (Holton et al., 209 2015) expressed under the maize ubiquitin promoter. We generated 10 independent 210 transgenic $T_{0}$ lines and inoculated the plants with Xoo using a leaf clipping method 211 followed by lesion length measurements, which allows a comparable but more rapid 212 assessment of resistance than bacterial population counting. We found that 8 of these 213 lines (lines 2, 3, 4, 5, 6, 7, 9, and 10) displayed enhanced resistance to Xoo compared 214 with the Kitaake parent line (Fig. S1).

215 To assess if the resistance phenotype was transmitted to the next generation, we 216 self-pollinated 5 of the $8 T_{0}$ lines (lines $2,4,5,6,7$ ) and collected $T_{1}$ seed. These $T_{1}$ 217 plants, as well as rice plants expressing and lacking Xa21 as controls, were inoculated 218 with $\mathrm{Xoo}$ and assessed for resistance by measuring the lengths of disease-induced 219 lesions. We observed that $T_{1}$ individuals that were PCR positive for the transgene in 220 lines 2,4,5, and 6 co-segregated with resistance to Xoo (PCR positive to negative ratios $2218: 4,21: 0,8: 7$, and 16:5, respectively). Lesion length averages were approximately $5 \mathrm{~cm}$ 222 in resistant individuals compared to approximately $13 \mathrm{~cm}$ for susceptible controls (Fig. 223 1, S2). All $T_{1}$ individuals from line 4 were PCR positive for Xa21:EFR:GFP (21:0) which 224 could have been from multiple transgene insertions $\left(X^{2}(1)=1.4, p=0.24\right)$ and were 225 resistant to $\mathrm{Xoo}_{\mathrm{o}}$. All $\mathrm{T}_{1}$ individuals from line 7 were also PCR positive for the 226 Xa21:EFR:GFP transgene. However, these plants showed varying degrees of 227 resistance (Fig. S2).

228 To determine if Xoo resistance in Xa21:EFR:GFP plants is mediated through 229 XA21 perception of RaxX, we infected two different T1 progeny from two different -2 230 lines (-2-13 and -2-19) and T2 progeny from line -6-5-1 with wild-type PXO99A, 
231 PXO99A $\Delta \operatorname{rax} X$ mutants $(\Delta \operatorname{rax} X)$ that evade full-length XA21-medaited immunity, and $232 \Delta \operatorname{rax} X$ strains complemented with $\operatorname{rax} X(\Delta \operatorname{rax} X(\operatorname{rax} X))$ that do no evade XA21-mediated

233 immunity (Pruitt et al., 2015). We found that Kitaake plants were susceptible to all

234 strains used (average lesion lengths approximately $20 \mathrm{~cm}$ ), whereas Xa21:GFP control 235 plants were susceptible to $\Delta r a x X$ infections with approximately $19 \mathrm{~cm}$ average lesion 236 lengths and resistant to WT PXO99A and complemented $\Delta \operatorname{rax} X(\operatorname{rax} X)$ strains with 237 approximately $7 \mathrm{~cm}$ average lesion lengths consistent with previous findings (Pruitt et 238 al., 2015). For segregants carrying the Xa21:EFR:GFP transgene, plants were significantly more resistant to WT PXO99A, with approximately $5 \mathrm{~cm}$ average lesion lengths, compared to $\Delta r a x X$ infections that developed lesions approximately $17 \mathrm{~cm}$ long. Xa21:EFR:GFP segregants carrying the transgene were significantly more resistant to $\Delta \operatorname{rax} X(\operatorname{rax} X)$ with approximately $8 \mathrm{~cm}$ average lesion lengths compared to nullsegregants that developed significantly longer average lesion lengths of approximately $17 \mathrm{~cm}$ (Fig. S3). These results indicate that the XA21:EFR:GFP protein perceives RaxX. For subsequent experiments, we focused on two Xa21:EFR:GFP lines (-2 and 6) for further molecular characterization. For these experiments, $T_{1}$ plants were used for line 2 and $T_{2}$ plants were used for line 6 (we self-pollinated $T_{1}$ individuals and collected $\mathrm{T}_{2}$ seed for line 6) to test if similar phenotypes are observable in different lines and in subsequent generations. We found that $T_{2}$ individuals from line 6 maintained $X_{o O}$ resistance that segregated with the Xa21:EFR:GFP transgene (Fig. 1). Because $T_{1}$ and

$251 \mathrm{~T}_{2}$ individuals from lines 2 and 6 , respectively, were still segregating for the transgene, 252 we performed experiments on individual plants that carried the Xa21:EFR:GFP 253 transgene, selected by PCR genotyping. We used null segregant individuals as 
254 controls.

255

256

257

258

259

260

261

262

263

264

265

266

267

268

269

270

271

272

273

274

275

276

The Xa21:EFR:GFP chimeric transgene is expressed and XA21:EFR:GFP protein accumulates in stable transgenic lines

We used qRT-PCR to assess if plants containing the Xa21:EFR:GFP transgene express the Xa21 ectodomain and EFR cytoplasmic domain. We assessed transcript levels using domain specific primers for regions that encode the XA21 ectodomain, XA21 cytoplasmic domain, and the EFR cytoplasmic domain (Table S1) (Fig. 2 a-c). Our results show Xa21:EFR:GFP-2-23, -2-24, -6-5-17 and -6-5-18 that carry the transgene specifically express regions encoding the XA21 ectodomain and the EFR cytoplasmic domain. Additionally, these plants do not express regions encoding the XA21 endodomain. Because these plants are not expressing the full-length Xa21 transcript or endodomain, any immune responses observed in these plants are not mediated by full-length XA21 or the XA21 endodomain.

In addition to the specific Xa21:EFR:GFP transcript, we show that XA21:EFR:GFP protein accumulates in transgenic rice. We performed Western Blot analysis to determine if XA21:EFR:GFP protein accumulates in Xa21:EFR:GFP transgenic rice using primary anti-GFP antibodies. Our results show that XA21:EFR:GFP protein is detectable in Xa21:EFR:GFP-2-28, -2-29, -6-5-4 and -6-5-7 that carry the Xa21:EFR:GFP transgene. Wild-type Kitaake and null segregants Xa21:EFR:GFP-2-32 and Xa21:EFR:GFP-6-5-6 do not express any GFP tagged protein (Fig. S3). Together, RNA and protein expression indicate that two independent Xa21:EFR:GFP transgenic lines express Xa21:EFR:GFP transcript and accumulate 
277 XA21:EFR:GFP protein.

278

279

280

RaxX21-sY treated Xa21:EFR:GFP rice leaves produce reactive oxygen species and highly express stress-related genes

We next assessed if Xa21:EFR:GFP rice are able to activate immune responses

after RaxX treatments. We used a commercially synthesized, sulfated RaxX peptide, 283

composed of 21 amino acids from the Xoo RaxX protein sequence in PXO99A

284

(RaxX21-sY) previously shown to activate XA21-mediated immunity (Pruitt et al., 2015;

285

Wei et al., 2016). Bursts of reactive oxygen species (ROS) are commonly measured to

assess immune responses because ROS are rapidly produced as a defense response

to pathogen attack (Wojtaszek, 1997; Jones \& Dangl, 2006; Macho \& Zipfel, 2014). We

therefore measured ROS production in Xa21:EFR:GFP rice after RaxX21-sY treatment

to determine if plants carrying the chimeric protein respond similarly to RaxX21-sY

290

treated plants carrying full-length XA21 (Pruitt et al., 2015). Xa21:EFR:GFP rice

291

accumulate ROS in response to RaxX21-sY treatments, but not to mock or elf18

treatments (Fig. 3). In addition, we confirmed that RaxX21-sY treated XA21:GFP rice,

expressing the full length XA21 protein tagged with GFP (Fig. S4), accumulate ROS

(Fig. 3 b). Null segregants did not produce ROS bursts in response to RaxX21-sY

treatments (Fig. 3). EFR:GFP and EFR:XA21:GFP rice responded to elf18, but not to

RaxX21-sY, showing that the XA21 ectodomain in full-length XA21 and XA21:EFR:GFP

proteins is necessary for RaxX-triggered immune responses (Fig. $3 \mathrm{c}$ and d).

We next measured stress-related marker gene expression in RaxX21-sY treated

Xa21:EFR:GFP rice to further characterize the XA21:EFR:GFP-mediated response. We 
300 measured the expression of rice defense marker genes PR10b, LOC_Os02g36190, 301 LOC_Os06g37224, and LOC_Os11g42200. PR10b encodes a putative ribonuclease 302 and is up-regulated upon fungal infection in rice (McGee \& Hamer, 2001),

303 LOC_Os02g36190 is involved in phytoalexin biosynthesis and bacterial blight resistance 304 (Li et al., 2013), LOC_Os06g37224 encodes an ent-Kaurene Oxidase up-regulated after 305 ultraviolet light stress (Itoh et al., 2004), and LOC_Os11g42200 encodes a laccase 306 precursor protein and is up-regulated after Atrazine herbicide treatment (Huang et al., 307 2016). The up-regulation of these genes were previously established as markers of the 308 rice stress-response including the XA21-mediated immune response to sulfated RaxX 309 peptides and to Xoo infection (Chen et al., 2014; Pruitt et al., 2015; Thomas et al., 2016) 310 using a detached leaf treatment assay. RNA was extracted from detached leaves of 4 311 week old plants mock treated with water or with $500 \mathrm{nM}$ of RaxX21-sY for 6 hours.

312 Gene expression was measured in individuals Xa21:EFR:GFP-2-23 and -6-5-17 by 313 quantitative real-time PCR. Higher expression was observed in each of the stress314 related genetic markers in RaxX21-sY treated Myc:Xa21 and Xa21:EFR:GFP rice (Fig. 3154 a-d). Gene expression was not induced in any mock treated samples or Kitaake 316 samples treated with RaxX21-sY. Only Individual 2-23 showed higher induction of 317 LOC_Os11g42200 and LOC_Os06g37224, and only induction of LOC_Os06g37224 318 was significant (Fig. 4 c). Although the magnitude of ROS and stress gene induction in 319 response to RaxX21-sY is relatively lower in Xa21:EFR:GFP rice compared to 320 XA21:GFP rice (Fig. 3 and Fig. 4), both show robust resistance to Xoo infection as 321 measured by lesion length progression (Fig. 1) Together, the results from ROS and 322 gene expression experiments suggest that the XA21 ectodomain in XA21:EFR:GFP is 
323 sufficient to recognize RaxX and that the EFR endodomain can be substituted for the

$324 \mathrm{XA21}$ endodomain to transduce immune responses after RaxX treatment.

325

326 Discussion

327 Here we show that the ectodomain of XA21 is sufficient to confer full resistance to Xoo

328 strain PXO99A when fused to the intracellular domain of the Arabidopsis immune

329 receptor EFR (Fig. 1, S1, S2, and S3). We previously demonstrated that a functional

330 EFR:XA21:GFP is not able to confer resistance to Xoo when expressed in rice.

331 Together these results suggest that the XA21 extracellular domain and the recognition

332 of $\operatorname{Rax} X$ are the key properties that dictate the robust immune response of XA21. Both

333 the native XA21 endodomain as well as the EFR endodomain fused to the XA21

334 ectodomain appear to be interchangeable as both XA21 and EFR kinases can confer

335 robust resistance when fused with the XA21 ectodomain. This result slightly contrasts

336 with previous domain swap studies that indicated that the endodomains of immune

337 receptors were the defining properties of the immune receptor responses (He et al.,

338 2000; Brutus et al., 2010; Albert et al., 2010; Kishimoto et al., 2010). Although XA21

339 mutants and XA21 derivatives that lack a functional kinase domain maintain partial

340 resistance, it appears that a functional kinase domain is required for robust resistance

341 (Wang et al., 1998; Andaya \& Ronald, 2003a).

342 Despite the evidence that rice expressing XA21:EFR:GFP are resistant to Xoo, it

343 is unclear why plants expressing the reciprocal EFR:XA21:GFP protein are susceptible

344 to $X_{0 o}$ (Schwessinger et al., 2015a). Previous results indicate that elf18 and RaxX21-sY

345 have similar $\mathrm{EC}_{50}$ values for immune activation (Schwessinger et al., 2015a; Pruitt et al., 
346 2015), but it is unclear if RaxX and EF-Tu are available at similar levels during infection,

347 and it is also possible that Xoo masks EF-Tu, preventing optimal EFR ectodomain

348 recognition. We hypothesize that the XA21 ectodomain is critical for conferring robust

349 resistance because it interacts with additional rice-specific signaling components that

350 the EFR ectodomain is unable to bind. In partial support of this hypothesis, we

351 previously showed that the EFR kinase domain does not interact with some of the

352 previously identified XA21 kinase domain signaling components, including the negative

353 regulator XB15 and positive regulator XB3 (Schwessinger et al., 2015a). It is therefore

354 possible that unidentified positive regulators of XA21-mediated immunity that interact

355 with the XA21 ectodomain do not associate with the EFR ectodomain. Future studies

356 might be aimed at identifying these elusive ectodomain specific signaling partners to

357 better understand XA21-mediated immunity.

358

359 Acknowledgments

360 We would like to thank Dr. Nicholas Holton and Prof. Dr. Cyril Zipfel from the Sainsbury

361 Laboratory for providing the Xa21:EFR:GFP construct. 


\section{References}

364

365

366

367

368

369

370

Albert M., Jehle AK., Mueller K., Eisele C., Lipschis M., Felix G. 2010. Arabidopsis thaliana pattern recognition receptors for bacterial elongation factor Tu and flagellin can be combined to form functional chimeric receptors. The Journal of Biological Chemistry 285:19035-19042.

Andaya CB., Ronald PC. 2003a. A Catalytically Impaired Mutant of Rice Xa21 Receptor Kinase Confers Partial Resistance to Xanthomonas oryzae pv. oryzae. Physiological and Molecular Plant Pathology 62:203-208.

Andaya CB., Ronald PC. 2003b. A catalytically impaired mutant of the rice Xa21 receptor kinase confers partial resistance to Xanthomonas oryzae pv oryzae. Physiological and Molecular Plant Pathology 62:203-208.

Brutus A., Sicilia F., Macone A., Cervone F., De Lorenzo G. 2010. A domain swap approach reveals a role of the plant wall-associated kinase 1 (WAK1) as a receptor of oligogalacturonides. Proceedings of the National Academy of Sciences of the United States of America 107:9452-9457.

Cabrera JC., Boland A., Messiaen J., Cambier P., Van Cutsem P. 2008. Egg box conformation of oligogalacturonides: the time-dependent stabilization of the elicitoractive conformation increases its biological activity. Glycobiology 18:473-482.

Chen X., Zuo S., Schwessinger B., Chern M., Daudi A., Canlas PE., Ruan DL., Zhou X., Wang J., Petzold C., Heazlewood J., Ronald PC. 2014. An XA21-associated kinase (OsSERK2) regulates immunity by the XA21 and XA3 immune receptors. Molecular Plant-microbe Interactions: MPMI doi: 10.1093/mp/ssu003. 
385 Chinchilla D., Bauer Z., Regenass M., Boller T., Felix G. 2006. The Arabidopsis receptor 386 kinase FLS2 binds flg22 and determines the specificity of flagellin perception. The $387 \quad$ Plant Cell 18:465-476.

388 Dardick C., Ronald P. 2006. Plant and animal pathogen recognition receptors signal $389 \quad$ through non-RD kinases. PLoS Pathogens 2:e2.

390 Dardick C., Schwessinger B., Ronald P. 2012. Non-arginine-aspartate (non-RD) kinases 391 are associated with innate immune receptors that recognize conserved microbial 392 signatures. Current Opinion in Plant Biology 15:358-366.

393 Decreux A., Messiaen J. 2005. Wall-associated kinase WAK1 interacts with cell wall 394 pectins in a calcium-induced conformation. Plant \& Cell Physiology 46:268-278.

395 Decreux A., Thomas A., Spies B., Brasseur R., Van Cutsem P., Messiaen J. 2006. In 396 vitro characterization of the homogalacturonan-binding domain of the wallassociated kinase WAK1 using site-directed mutagenesis. Phytochemistry

Felix G., Duran JD., Volko S., Boller T. 1999. Plants have a sensitive perception system 400 for the most conserved domain of bacterial flagellin. The Plant Journal: for Cell and

Ferrari S., Galletti R., Pontiggia D., Manfredini C., Lionetti V., Bellincampi D., Cervone 403 Molecular Biology 18:265-276.

Gómez-Gómez L., Boller T. 2000. FLS2: An LRR Receptor-like Kinase Involved in the 407 Perception of the Bacterial Elicitor Flagellin in Arabidopsis. Molecular Cell 5:1003- 
409

410

411

412

413

414

415

416

417

418

419

420

421

422

423

424

425

426

427

428

429

430

He Z., Wang ZY., Li J., Zhu Q., Lamb C., Ronald PC., Chory J. 2000. Perception of brassinosteroids by the extracellular domain of the receptor kinase BRI1. Science 288:2360-2363.

Hiei Y., Ohta S., Komari T., Kumashiro T. 1994. Efficient transformation of rice (Oryza sativa L.) mediated by Agrobacterium and sequence analysis of the boundaries of the T-DNA. The Plant Journal: for cell and molecular biology 6:271-282.

Holton N., Nekrasov V., Ronald PC., Zipfel C. 2015. The Phylogenetically-Related Pattern Recognition Receptors EFR and XA21 Recruit Similar Immune Signaling Components in Monocots and Dicots. PLoS Pathogens 11:e1004602.

Huang MT., Lu YC., Zhang S., Luo F., Yang H. 2016. Rice (Oryza sativa) Laccases Involved in Modification and Detoxification of Herbicides Atrazine and Isoproturon Residues in Plants. Journal of agricultural and food chemistry 64:6397-6406.

Itoh H., Tatsumi T., Sakamoto T., Otomo K., Toyomasu T., Kitano H., Ashikari M., Ichihara S., Matsuoka M. 2004. A rice semi-dwarf gene, Tan-Ginbozu (D35), encodes the gibberellin biosynthesis enzyme, ent-kaurene oxidase. Plant molecular biology 54:533-547.

Jones JDG., Dangl JL. 2006. The plant immune system. Nature 444:323-329.

Kaku H., Nishizawa Y., Ishii-Minami N., Akimoto-Tomiyama C., Dohmae N., Takio K., Minami E., Shibuya N. 2006. Plant cells recognize chitin fragments for defense signaling through a plasma membrane receptor. Proceedings of the National Academy of Sciences of the United States of America 103:11086-11091.

Kauffman HE., Reddy APK., Hsiek SPV., Marca SD. 1973. An improved technique for 
431

432

433

434

435

436

437

438

439

440

441

442

443

444

445

446

447

448

449

450

451

452

453

evaluating resistance of race varieties to Xanthomonas oryzae. Plant Disease Management Reports 57:537-541.

Kishimoto K., Kouzai Y., Kaku H., Shibuya N., Minami E., Nishizawa Y. 2010.

Perception of the chitin oligosaccharides contributes to disease resistance to blast fungus Magnaporthe oryzae in rice. The Plant journal: for Cell and Molecular Biology 64:343-354.

Kunze G., Zipfel C., Robatzek S., Niehaus K., Boller T., Felix G. 2004. The N Terminus of Bacterial Elongation Factor Tu Elicits Innate Immunity in Arabidopsis Plants. The Plant Cell.

Li J., Chory J. 1997. A putative leucine-rich repeat receptor kinase involved in brassinosteroid signal transduction. Cell 90:929-938.

Li W., Shao M., Yang J., Zhong W., Okada K., Yamane H., Qian G., Liu F. 2013. Oscyp71Z2 involves diterpenoid phytoalexin biosynthesis that contributes to bacterial blight resistance in rice. Plant science: an international journal of experimental plant biology 207:98-107.

Livak KJ., Schmittgen TD. 2001. Analysis of relative gene expression data using realtime quantitative PCR and the 2(-Delta Delta C(T)) Method. Methods 25:402-408.

Macho AP., Zipfel C. 2014. Plant PRRs and the activation of innate immune signaling. Molecular Cell 54:263-272.

McGee JD., Hamer JE. 2001. Characterization of a PR-10 pathogenesis-related gene family induced in rice during infection with Magnaporthe grisea. Molecular plantmicrobe interactions: MPMI.

Pruitt RN., Schwessinger B., Joe A., Thomas N., Liu F., Albert M., Robinson MR., Chan 
454

455

456

457

458

459

460

461

462

463

464

465

466

467

468

469

470

471

472

473

474

475

476

LJG., Luu DD., Chen H., Bahar O., Daudi A., De Vleesschauwer D., Caddell D., Zhang W., Zhao X., Li X., Heazlewood JL., Ruan D., Majumder D., Chern M., Kalbacher H., Midha S., Patil PB., Sonti RV., Petzold CJ., Liu CC., Brodbelt JS., Felix G., Ronald PC. 2015. The rice immune receptor XA21 recognizes a tyrosinesulfated protein from a Gram-negative bacterium. Science Advances 1:e1500245.

Ronald PC., Beutler B. 2010. Plant and animal sensors of conserved microbial signatures. Science 330:1061-1064.

Schwessinger B., Bahar O., Thomas N., Holton N., Nekrasov V., Ruan D., Canlas PE., Daudi A., Petzold CJ., Singan VR., Kuo R., Chovatia M., Daum C., Heazlewood JL., Zipfel C., Ronald PC. 2015a. Transgenic Expression of the Dicotyledonous Pattern Recognition Receptor EFR in Rice Leads to Ligand-Dependent Activation of Defense Responses. PLoS Pathogens 11:e1004809.

Schwessinger B., Li X., Ellinghaus TL., Chan LJG., Wei T., Joe A., Thomas N., Pruitt R., Adams PD., Chern MS., Petzold CJ., Liu CC., Ronald PC. 2015b. A secondgeneration expression system for tyrosine-sulfated proteins and its application in crop protection. Integrative Biology 8:542-545.

Song WY., Wang GL., Chen LL., Kim HS., Pi LY., Holsten T., Gardner J., Wang B., Zhai WX., Zhu LH., Fauquet C., Ronald P. 1995. A receptor kinase-like protein encoded by the rice disease resistance gene, Xa21. Science 270:1804-1806.

Takai R., Isogai A., Takayama S., Che F-S. 2008. Analysis of flagellin perception mediated by flg22 receptor OsFLS2 in rice. Molecular Plant-microbe Interactions: MPMI 21:1635-1642.

Thomas NC., Schwessinger B., Liu F., Chen H., Wei T., Nguyen YP., Shaker IWF., 
477 Ronald PC. 2016. XA21-specific induction of stress-related genes following

$478 \quad$ Xanthomonas infection of detached rice leaves. PeerJ 4:e2446.

479 Wang GL., Ruan DL., Song WY., Sideris S., Chen L., Pi LY., Zhang S., Zhang Z.,

480 Fauquet C., Gaut BS., Whalen MC., Ronald PC. 1998. Xa21D encodes a receptor-

481 like molecule with a leucine-rich repeat domain that determines race-specific

482 recognition and is subject to adaptive evolution. The Plant Cell 10:765-779.

483 Wei T., Chern M., Liu F., Ronald PC. 2016. Suppression of bacterial infection in rice by

484 treatment with a sulfated peptide. Molecular Plant Pathology. DOI:

$485 \quad 10.1111 / \mathrm{mpp} .12368$.

486 Wojtaszek P. 1997. Oxidative burst: an early plant response to pathogen infection.

$487 \quad$ Biochemical Journal $322($ Pt 3):681-692.

488 Zipfel C., Kunze G., Chinchilla D., Caniard A., Jones JDG., Boller T., Felix G. 2006.

489 Perception of the bacterial PAMP EF-Tu by the receptor EFR restricts

$490 \quad$ Agrobacterium-mediated transformation. Cell 125:749-760. 
492

493 Figures

A

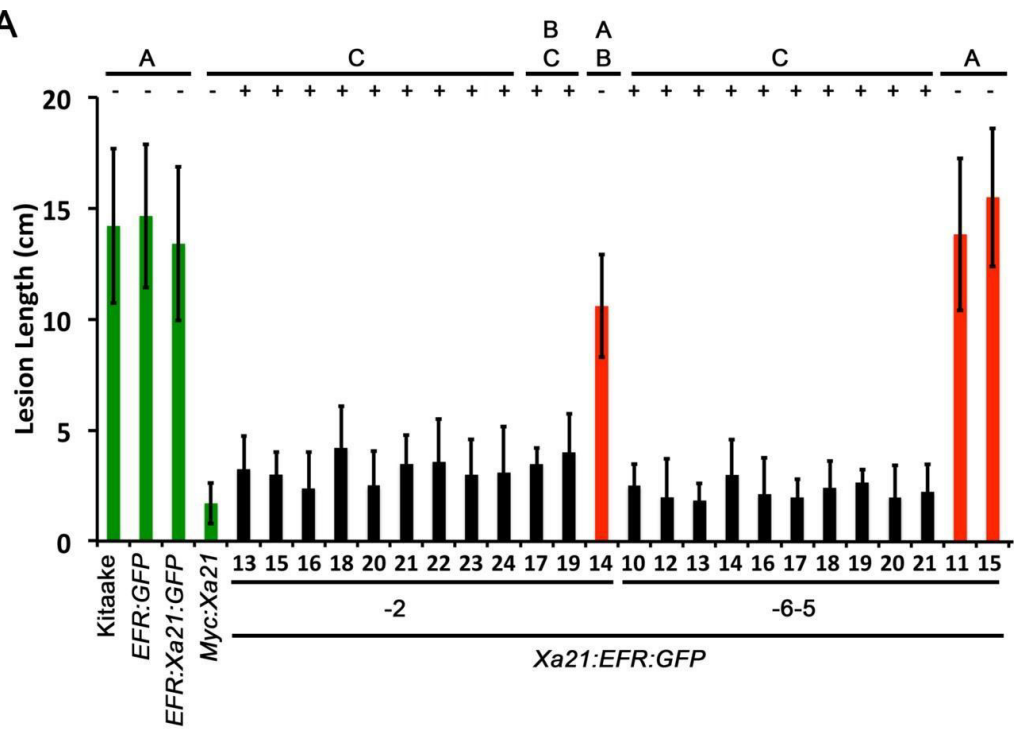

494

495

496

497

498

499

500

501

502

503

504

505

506

507

508

509

510
B

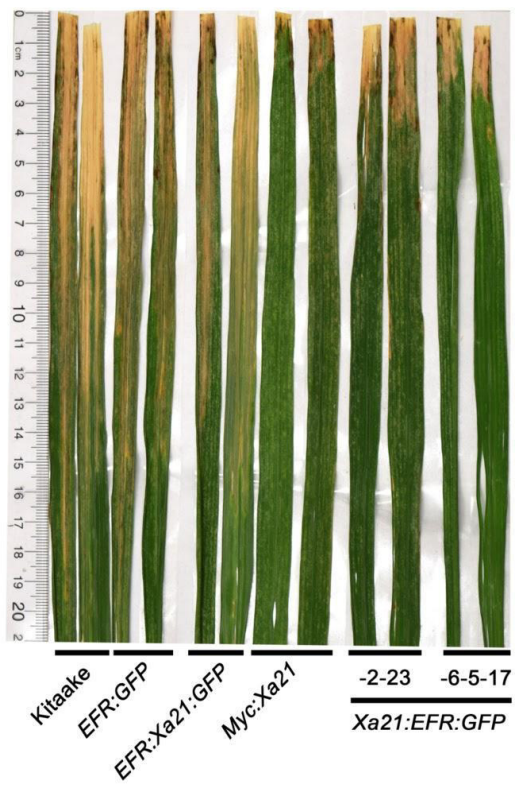

Figure 1. Rice expressing Xa21:EFR:GFP are resistant to Xoo infection

$A$, The bar graph represents the average lesion length observed on rice plants infected with Xoo. Control lines used were Kitaake, EFR:GFP, EFR:XA21:GFP, and Myc:XA21 rice (green bars). Experimental samples include individuals $P C R$ positive for the Xa21:EFR:GFP transgene (black bars) from line 2 and 6 and PCR negative individuals (red bars). Five week old greenhouse grown plants were scissor inoculated with

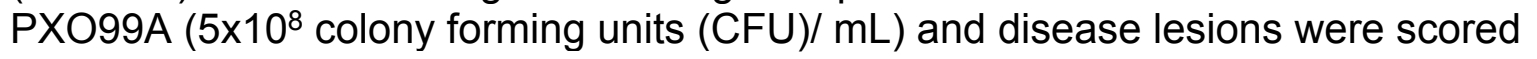
approximately 2 weeks post inoculation. Error bars represent standard deviation from the mean lesion length. Mean lesion lengths are the average of lesion measurements from individual leaves from the same plant $(n \geq 3)$. Black lines and letters above the graph represent statistical groupings using the Tukey-Kramer HSD test. Different letters indicate significant differences $(p$ $<0.05)$. This experiment was repeated at least three times with similar results. B, Photograph of select leaves from the same experiment in A. The photograph shows Kitaake, EFR:GFP, EFR:Xa21:GFP, Myc:Xa21, Xa21:EFR:GFP individual -2-23, and -6-5-17 leaves infected with $X o o$ and was taken approximately 2 weeks after inoculation. Nicholas Thomas provided the photograph. 
511

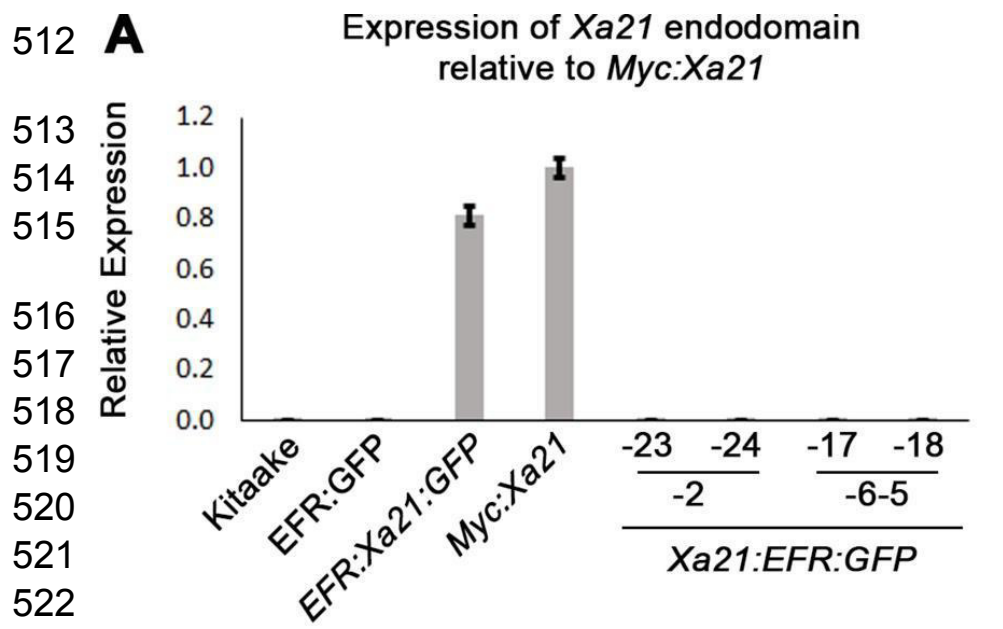

523

524

525

526

527

528

529

530

531

532

533

534
Figure 2. Xa21:EFR:GFP transcripts are expressed in stable transgenic lines

Bar graphs represent the relative expression of transgenic transcripts. A, relative amplification of the Xa21

endodomain with Myc:Xa21 rice as the expression reference. $B$, Amplification of the Xa21 endodomain with Myc:Xa21 rice as the expression reference. $\mathrm{C}$, Amplification of the EFR cytoplasmic domain with EFR:GFP rice as the expression reference. Gene expression was measured by quantitative real-time PCR using cDNA amplified from total RNA as a template. Each gene expression measurement is the average of 2 technical replicates and error bars represent the standard deviation between the two measurements.

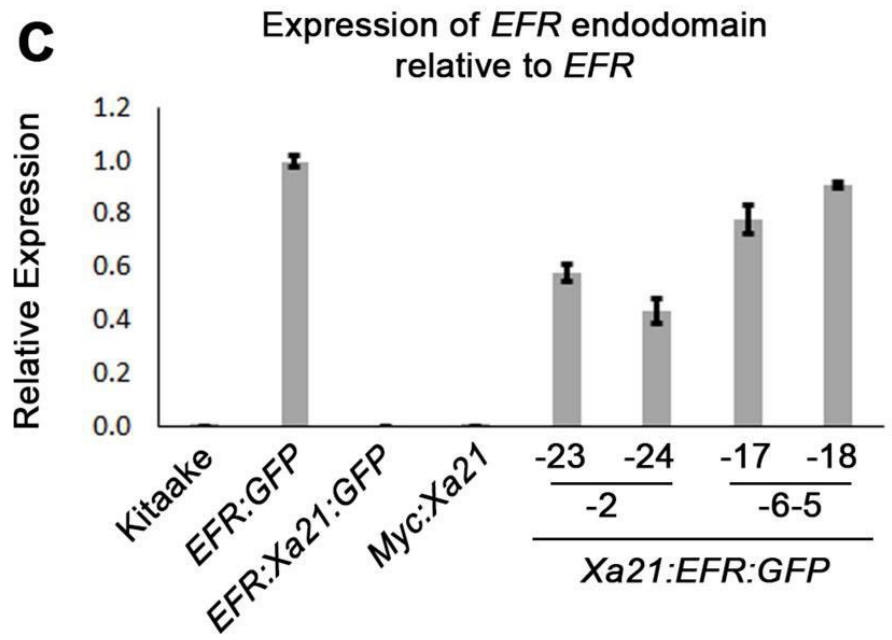



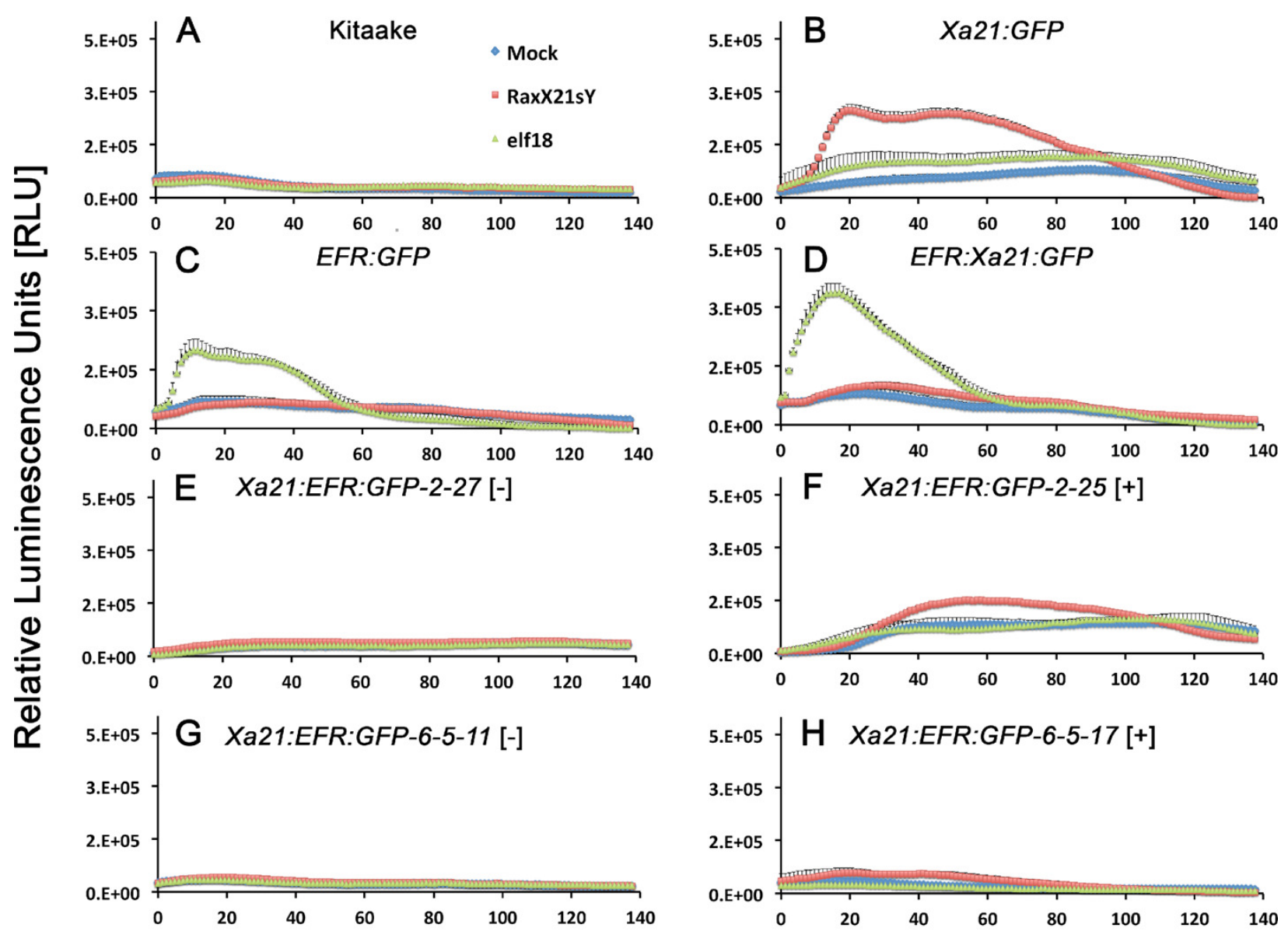

535

\section{6}

537

538

539

540

541

542

543

544

545

\section{Time [m]}

Figure 3. Reactive oxygen species accumulate after peptide treatments

Reactive oxygen species (ROS) production after water (mock, blue diamonds), $500 \mathrm{nM}$ RaxX21-sY (red squares), or $500 \mathrm{nM}$ elf18 peptide treatments (green triangles). A, ROS production in wild-type Kitaake rice and $B$, Xa21:GFP rice C, EFR:GFP rice and D, EFR:Xa21:GFP rice. $E$ and $G$, ROS production in T1 and T2 null-segregant individuals from Xa21:EFR:GFP line -2 and line $-6-5$, respectively. $F$ and $H$, ROS production in $T 1$ and T2 ndividuals from line -2 and $-6-5$, respectively, that segregate for the Xa21:EFR:GFP transgene. Each datapoint represents an average of four technical replicate measurements and error bars represent the standard error of the averages. These experiments have been repeated three times with similar results. 
A PR10b

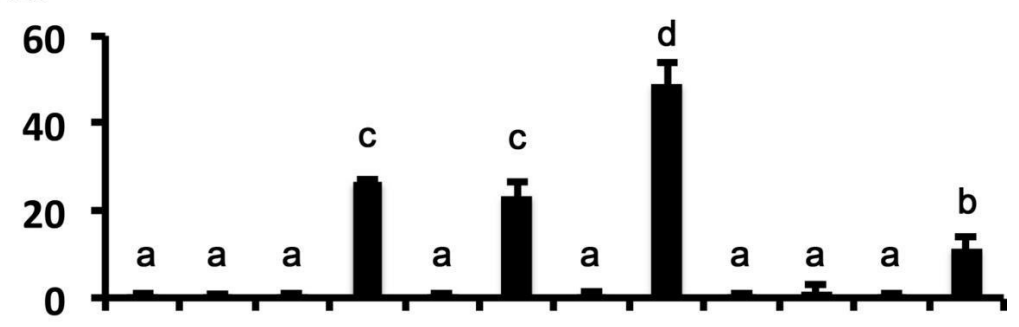

B LOC_Os02g36190

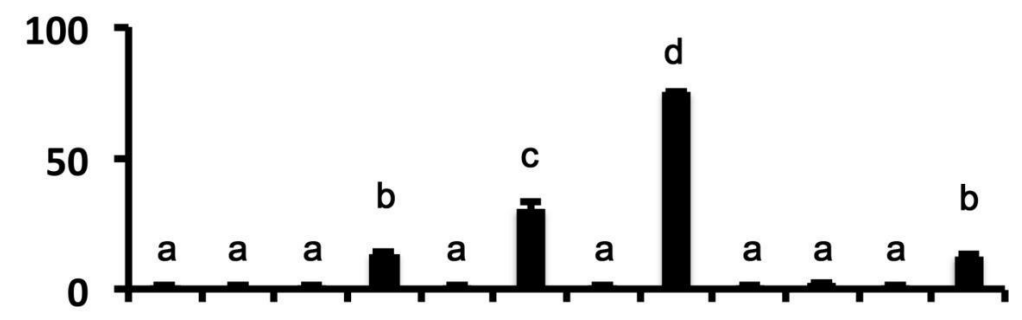

C LOC_Os06g37224

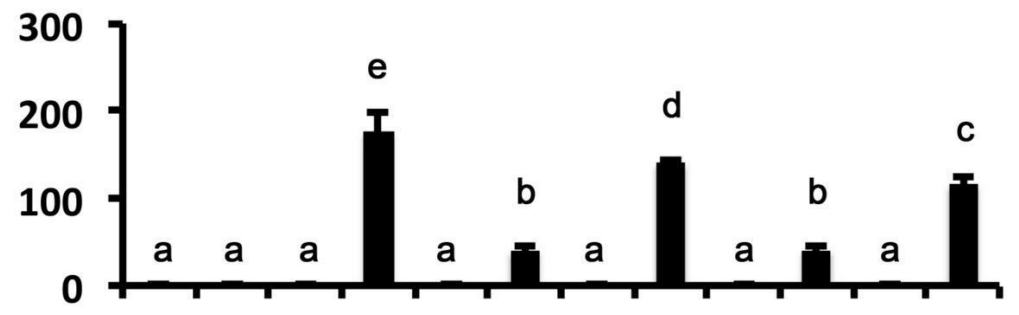

D LOC_Os11g42200

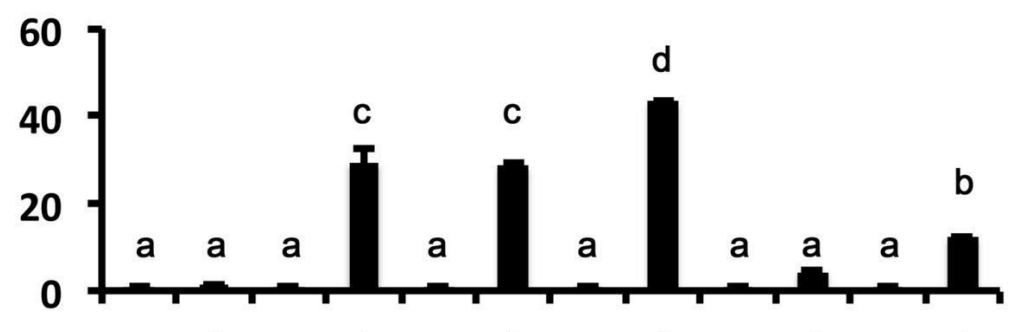

Treatment

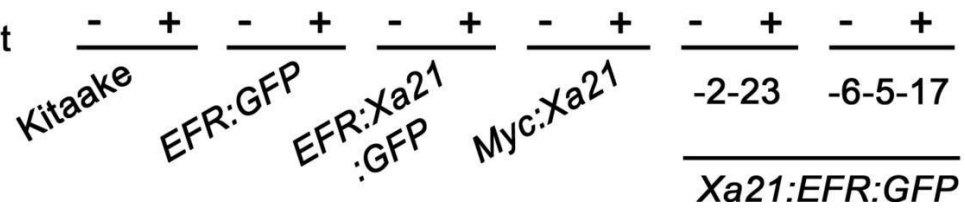

548 Figure 4. Xa21:EFR:GFP rice express stress related genes after RaxX21-sY 549 treatment

550 Gene expression profiles of four stress-related genes, PR10b, LOC_Os2g36190, 551 LOC_06g37224, and LOC_Os11g42200. Samples are rice leaves from wild-type 552 Kitaake, Myc:XA21 rice, and individuals -23 from Xa21:EFR:GFP line -2 and individual 55317 from line -6-5. Leaves were mock treated with water (-) or with $500 \mathrm{nM}$ RaxX21-sY 554 for Myc:XA21 and XA21:EFR rice and 500nM elf18 for EFR:GFP and EFR:XA21:GFP 555 rice (+). Kitaake was treated with both RaxX21-sY and elf18 in these experiments. 
556 Letters indicate significant difference in gene expression compared to mock using the 557 Tukey-Kramer HSD test (alpha $=0.05$ ). Expression levels are normalized to mock 558 treatment of the same line. Bars depict average expression level relative to actin 559 expression \pm standard error of three technical replicates. This experiment was repeated 560 twice with similar results. 